\title{
Correction: EZH2 targeting reduces medulloblastoma growth through epigenetic reactivation of the BAI1/p53 tumor suppressor pathway
}

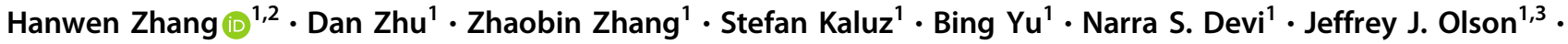 \\ Erwin G. Van Meir $\mathbb{1}^{1,3,4,5}$
}

Published online: 1 November 2019

(c) The Author(s), under exclusive licence to Springer Nature Limited 2019

\section{Correction to: Oncogene}

https://doi.org/10.1038/s41388-019-1036-7

The original version of this Article contained an error in the author affiliations.

Affiliation number 1 incorrectly read 'Department of Neurosurgery, Wallace Tumor Institute, University of Alabama at Birmingham, 520E, 1720 2nd Ave. South, Birmingham, AL 35294, USA'. It should be 'Laboratory of Molecular Neuro-Oncology, Department of Neurosurgery and Hematology \& Medical Oncology, School of Medicine, Emory University, Atlanta, GA, 30322, USA'. A new affiliation has been added for the Author Erwin G. Van Meir, which is 'Department of Neurosurgery and O'Neal Comprehensive Cancer Center, University of Alabama at Birmingham (UAB), WTI 520E, 1720 2nd Ave. South Birmingham, AL, 35294, USA'.

This has now been corrected in both the PDF and HTML versions of the Article.
These authors contributed equally: Hanwen Zhang, Dan Zhu

The original article can be found online at https://doi.org/10.1038/ s41388-019-1036-7.

Erwin G. Van Meir

evanmeir@uab.edu

1 Laboratory of Molecular Neuro-Oncology, Department of Neurosurgery and Hematology \& Medical Oncology, School of Medicine, Emory University, Atlanta, GA 30322, USA

2 Department of Neurosurgery, Xiangya Hospital and School of Medicine, Central South University, Changsha 410013 Hunan, PR China

3 Winship Cancer Institute, Emory University, Atlanta, GA 30322, USA

4 Department of Hematology \& Medical Oncology, School of Medicine, Emory University, Atlanta, GA 30322, USA

5 Department of Neurosurgery and O'Neal Comprehensive Cancer Center, University of Alabama at Birmingham (UAB), WTI 520E, 1720 2nd Ave., South Birmingham, AL 35294, USA 